Julia Hauck

\title{
Eine für alle?! Leichte Sprache als Alternative zur Mehrsprachigkeit
}

\begin{tabular}{ll}
\hline Kurzbeschreibung & $\begin{array}{l}\text { Leichte Sprache ist eine Sonderform des Standarddeutschen, wel- } \\
\text { che durch einen einfachen Satzbau und reduzierten Wortschatz } \\
\text { besonders leicht verständlich ist. } \\
\text { Leichte Sprache wurde von und mit Menschen mit Behinderung } \\
\text { entwickelt, um den Zugang zu Informationen zu erleichtern und } \\
\text { damit Teilhabe zu ermöglichen. }\end{array}$ \\
\hline Zielgruppe & - Menschen mit geistiger Behinderung \\
& - Menschen mit Hör- oder Sehbehinderung \\
& - Menschen mit Aphasie \\
& - Ältere mit beginnender Demenz \\
& - Funktionale Analphabeten \\
& - Menschen mit geringen Deutschkenntnissen \\
\hline Anwendungsgebiete & - Informationsmaterialien in/über die Bibliothek \\
& - Öffentlichkeitsarbeit, externe Kommunikation \\
& - Führungen \& Veranstaltungen \\
& - Bestandsaufbau: Easy Reader \\
\hline Kritik & - Vereinfachung/Verkürzung des Inhalts \\
& - Stigmatisierung von Zielgruppen \\
& - Vorwurf inkorrekter Rechtschreibung und Grammatik \\
\hline
\end{tabular}

Abb. 21: Übersicht zum Thema „Leichte Sprache“

Im Positionspapier von dbv und CILIP zum Thema „Bibliotheken und die Diversität in der Gesellschaft“ (2011) wird von Bibliotheken gefordert, sich an der „Vielfalt und den Bedürfnissen der Einwohnerinnen und Einwohner der Kommune“ $\mathrm{zu}$ orientieren sowie deren „Kenntnisse, Interessen sowie Anforderungen“ einzubeziehen. Diese Forderungen bezogen sich damals „insbesondere auf in jüngster Zeit angekommene Migranten, Flüchtlinge und Asylbewerber, lokal, national und international nur vorübergehend sesshafte Personen sowie ethnische Minderheiten“. Gemeint ist hier eine interkulturelle Bibliotheksarbeit, die ihren Fokus auf Menschen mit (familiärer) Migrationserfahrung und seit 2015 vermehrt direkt auf Geflüchtete in Deutschland legt. Im Mittelpunkt stehen mehrsprachige Materialien/Informationen sowie der Bestandsaufbau von Literatur in verschiedenen Sprachen (vgl. Deutscher Bibliotheksverband e.V., 2017). Hermann Rösch (2013, S. 13) verweist diesbezüglich auf Medienangebote 
in verschiedenen Muttersprachen und Veranstaltungen zur Herkunftskultur, Sprachkurse und andere Medien zum Deutschlernen sowie Material zur sogenannten „deutschen Gesellschaft und Kultur“. Zum einen sollen Menschen dabei unterstützt werden, ihre Deutschkenntnisse zu verbessern. Zum anderen sollen Mehrsprachigkeit und kulturelle Vielfalt wertschätzend in die Angebote der Bibliothek integriert werden.

Doch wie lässt sich dieser Anspruch in der Realität umsetzen ${ }^{1}$ Nimmt man beispielsweise Jena als eine durchschnittliche kleine Großstadt (etwas weniger als 110000 Einwohner*innen) im Osten Deutschlands mit lediglich 14\% Menschen mit Migrationshintergrund (Migrationsbericht Jena, 2019), so werden neben Deutsch auch geschätzt mindestens 20 verschiedene Muttersprachen gesprochen. Setzt man die sprachliche und informationelle Teilhabe aller Menschen als Ziel, so müsste die dort ansässige Ernst-Abbe-Bücherei Jena Informationsangebote und Medien in zig Sprachen vorrätig haben. Die personellen, finanziellen oder auch räumlichen Ressourcen für Übersetzungsleistungen und mehrsprachigen Bestand sind jedoch begrenzt, sodass nicht alle (potenziellen) Nutzer`innen gleichermaßen in (einer) ihrer Muttersprachen angesprochen werden können.

Beschränkt man sich gezwungenermaßen auf die zahlenmäßige Top Ten der (angenommenen) Muttersprachen-Gruppen in Jena, so bleibt immerhin noch die Aufgabe, Arabisch, Chinesisch, Russisch, Englisch/Hindi, Persisch, Ukrainisch, Italienisch, Kurdisch, Vietnamesisch und Rumänisch anzubieten. Dabei sind die Schul-/Weltsprachen Französisch, Spanisch und Portugiesisch noch nicht inkludiert. Die sprachliche Vielfalt der Stadtgesellschaft stellt eine Bibliothek - in Jena und auch anderenorts - vor eine Herkules-Aufgabe: Wie ermöglichen wir mit unseren Ressourcen allen, wirklich allen, einen gerechten Zugang zu Informationen sowie adäquate Medien-Angebote?

Hier hilft es, einen Schritt zurückzugehen: Deutsch ist offiziell Amtssprache in der Verwaltung der Bundesrepublik ( $\$ 23 \mathrm{BVwVfG}$ ) und so kommt man bei Behörden, Bildungseinrichtungen und im Alltag nicht umher, mündlich und schriftlich in deutscher Sprache zu kommunizieren. Hier sind Menschen, die geringe Deutschkenntnisse vorweisen, im Nachteil. Doch dies ist bei Weitem kein Alleinstellungsmerkmal von Geflüchteten, Ausländer^innen oder Menschen mit Migrationshintergrund. Auch Menschen mit sogenannten körperlichen und geistigen Behinderungen und/oder schweren Erkrankungen ringen seit Jahrzehnten um sprachliche Teilhabe am gesellschaftlichen Dasein. „Deutsche

1 Leichte Sprache ist Teil einer Strategie der inklusiven Sprache in Bibliotheken, deren Ziel es ist, auf vielfältige Weise kommunikative Barrieren für diverse Zielgruppen abzubauen (vgl. Hotze, 2021). 
Sprache, schwere Sprache“ gilt für deutlich mehr Menschen, als Otto-NormalVerbraucher`in traditionell in den Sinn kommen. Ein zeitgemäßes Mittel, um kommunikative Teilhabe zu ermöglichen, ist das Konzept der Leichten Sprache:

Leichte Sprache wurde ursprünglich von Selbstvertreterinnen und Selbstvertretern entwickelt, die sich als Menschen mit Lernschwierigkeiten beschreiben. Die Idee bestand zunächst darin, speziell dieser Zielgruppe Zugang zu wichtigen Informationen zu verschaffen. Dabei ging es auf der Zielebene unter anderem darum, mittels Leichter Sprache die eigenen Rechte besser zu kennen, um sie wirkungsvoll vertreten zu können und somit ein Instrument der Selbstvertretung zur Verfügung zu haben. (Seitz, 2014, S. 4)

\section{Die Zielgruppen}

Der Ursprung der Leichten Sprache geht auf die amerikanische Organisation People First zurück, die sich seit Mitte der 1970er-Jahre für die Belange von Menschen mit Behinderung einsetzt und in den Neunzigern die Idee des Easy Read entwickelte. In Deutschland veröffentlichte der Verein Mensch zuerst ab 2001 zwei Wörterbücher zur Leichten Sprache. 2006 folgte die Etablierung des Netzwerks Leichte Sprache (vgl. Kellermann, 2014). Wie viele Menschen in der Bundesrepublik Schwierigkeiten beim Verständnis von geschriebener Sprache haben, ist schwer zu erfassen:

Denn Literalität ist relational. Die Anforderungen an Schriftsprachlichkeit sind historisch und kulturell variabel. Sie haben sich in den vergangenen Jahren insbesondere durch die Verbreitung digital verarbeiteter Schrift deutlich erhöht. Der gesellschaftliche Wandel kann somit als ein Bedingungsfaktor des funktionalen Analphabetismus beschrieben werden. (Nickel, 2014, S. 27)

Die LEO-Studie 2018 (Grotlueschen et al., 2018) der Universität Hamburg, die vom Bundesministerium für Bildung und Forschung gefördert wurde, zeigt, dass rund 6,2 Millionen Erwachsene in Deutschland nur eine geringe Literalität vorweisen. Damit gelten rund 12,1\% der Deutsch sprechenden Bevölkerung zwischen 18 und 64 Jahren als sogenannte funktionale Analphabeten ${ }^{2}$. Laut der

2 „Funktionaler Analphabetismus ist gegeben, wenn die schriftsprachlichen Kompetenzen von Erwachsenen niedriger sind als diejenigen, die minimal erforderlich sind und als selbstverständlich vorausgesetzt werden, um den jeweiligen gesellschaftlichen Anforderungen gerecht $\mathrm{zu}$ werden. [...] Dies ist gegenwärtig zu erwarten, wenn eine Person nicht in der Lage ist, aus einem einfachen Text eine oder mehrere direkt enthaltene Informationen sinnerfassend $\mathrm{zu}$ lesen und/oder sich beim Schreiben auf einem vergleichbaren Kompetenzniveau befindet.“ (Egloff et al., 2011, S. 14 f.). In der LEO-Studie (Grotlueschen et al., 2018, S. 5) wird empfohlen, eher den Begriff „geringe Literalität“ bzw. „gering literalisierte Erwachsene“ zu nutzen. 
Studie können 4,4\% der Bevölkerung maximal Wörter, aber keine Sätze lesen. Weitere acht Prozent können nur kurze Sätze, aber keine gesamten Texte erfassen. Neben diesen funktionalen Analphabeten gibt es außerdem eine große Gruppe an Menschen, die erhebliche Probleme beim Schreiben und Lesen haben, d.h. deren verschriftlichter Wortschatz Grundschulniveau ähnelt. Dies trifft laut der LEO-Studie auf ganze 10,6 Millionen erwachsene Menschen $(20,5 \%)$ in Deutschland zu.

Hier zeigt sich, dass der Einsatz von Leichter Sprache nicht nur einer kleinen Minderheit dient: Knapp ein Drittel (32,6\%) der deutschen Bevölkerung kann nicht richtig lesen oder schreiben. Zwar machen Personen, die Deutsch als Zweitsprache gelernt haben, einen großen Anteil (47,4\%) davon aus, jedoch sind über die Hälfte (52,6\%) der Betroffenen Deutsch-Muttersprachler^innen.

Die Vielfalt derer, die von Leichter Sprache profitieren, ist groß (vgl. Stefanowitsch, 2014): Sowohl Menschen mit Lernbehinderungen/-einschränkungen und anderen kognitiven Einschränkungen wie auch Menschen, deren Muttersprache nicht Deutsch ist - einschließlich Gebärdender - gehören dazu. Aber auch älteren Menschen mit beginnender Demenz kann Leichte Sprache helfen. Ausführliche Informationen zu den einzelnen Zielgruppen finden sich in den Beschreibungen von Ursula Bredel und Christiane Maaß (2015, S. 40 ff.). Für die einen dient Leichte Sprache als Kommunikationsmodus Nr. 1 und für die anderen als Brücke, um den Übergang zur Standard-Schriftsprache zu begleiten. $\mathrm{Zu}$ dem hilft Leichte Sprache sekundär auch den Mittler`innen, die mit den sogenannten Zielgruppen arbeiten, leben und/oder interagieren. Dazu zählen im weitesten Sinne auch die Mitarbeitenden in Öffentlichen Bibliotheken, um adäquate Medien bereitzustellen und mit diesen Nutzer^innen möglichst barrierefrei kommunizieren zu können.

\section{Die Regeln}

Für Leichte Sprache gibt es unterschiedliche Regelwerke und Empfehlungen: Das Netzwerk Leichte Sprache hat beispielsweise 17 essenzielle Regeln aus der Praxis heraus entwickelt (Bredel \& Maaß, 2015, S. 30-31). Eine umfassende Zusammenfassung der verschiedenen Regeln sowie Hinweise für eine leichte Lesbarkeit finden sich im Ratgeber des Bundesministeriums für Arbeit und Soziales in Zusammenarbeit mit dem Netzwerk Leichte Sprache (Leichte Sprache. Ein Ratgeber., 2014): 
- Benutzen Sie einfache Wörter, z. B. „erlauben“ statt „genehmigen“.

- Benutzen Sie Wörter, die etwas genau beschreiben, z. B. „Bus und Bahn“ statt „öffentlicher Nahverkehr“.

- Benutzen Sie bekannte Wörter. Verzichten Sie auf Fach-Wörter und FremdWörter, z. B. „Arbeits-Gruppe“ statt „Workshop“.

- Benutzen Sie immer die gleichen Wörter für die gleichen Dinge, z. B. immer „Tablette“ statt auch mal „Pille“.

- Benutzen Sie kurze Wörter, z. B. „Bus“ statt „Omnibus“.

- Verzichten Sie auf Abkürzungen, z. B. „das heißt“ statt „d. h.“.

- Benutzen Sie Verben, z. B. „Wir wählen morgen.“ statt „Die Wahl ist morgen.“

- Benutzen Sie aktive Wörter, z. B. „Wir wählen morgen.“ statt „Morgen wird gewählt.“

- Vermeiden Sie den Genitiv, z. B. „das Haus vom Lehrer“ statt „des Lehrers Haus“.

- Vermeiden Sie den Konjunktiv, z. B. „Es regnet vielleicht.“ statt „Es könnte regnen."

- Benutzen Sie positive Sprache, z. B. „Ich bin krank.“ statt „Ich bin nicht gesund."

- Vermeiden Sie Redewendungen und bildliche Sprache, z. B. „schlechte Eltern“ statt „Rabeneltern“.

- Schreiben Sie Zahlen so, wie die meisten Menschen sie kennen, z. B. „9“ statt „IX“.

- Vermeiden Sie alte Jahreszahlen, z. B. „vor mehr als 100 Jahren“ statt „1765“.

- Vermeiden Sie hohe Zahlen und Prozentzahlen, z. B. „viele Menschen“ statt „13432 Menschen“.

- Schreiben Sie Zahlen als Ziffern, z. B. „5 Frauen“ statt „fünf Frauen“.

- Vermeiden Sie Sonderzeichen, z. B. „und“ statt „\&“.

- Schreiben Sie kurze Sätze. Machen Sie in jedem Satz nur eine Aussage, z. B. „Ich kann Ihnen helfen. Sagen Sie mir: Was wünschen Sie?“ statt „Wenn Sie mir sagen, was Sie wünschen, kann ich Ihnen helfen.“

- Benutzen Sie einen einfachen Satzbau, z. B. „Wir fahren zusammen in den Urlaub.“ statt „Zusammen fahren wir in den Urlaub.“

- Am Anfang vom Satz dürfen auch stehen: oder, wenn, weil, und, aber.

- Sprechen Sie die Leser^in persönlich an: „Sie dürfen morgen wählen.“ statt „Morgen ist die Wahl.“

- Benutzen Sie die Anrede „Sie“.

- Vermeiden Sie Fragen im Text - als Überschrift sind sie aber sinnvoll. 
- Benutzen Sie eine einfache serifenlose Schrift mit mindestens Schriftgröße 14.

- Lassen Sie genügend Abstand zwischen den Zeilen.

- Schreiben Sie immer linksbündig.

- Schreiben Sie jeden neuen Satz in eine neue Zeile.

- Trennen Sie keine Wörter am Ende einer Zeile.

- Machen Sie viele Absätze und Überschriften.

- Heben Sie wichtige Dinge hervor - am besten fett markiert, nicht kursiv oder unterstrichen.

- Benutzen Sie dunkle Schrift und helles Papier.

- Benutzen Sie dickes, mattes Papier.

- Benutzen Sie (scharfe und klare) Bilder - aber nicht als Hintergrund.

Unterschiedliche Ansichten existieren zum Thema Gliederung von langen Wörtern, ob nun durch einen Bindestrich oder Mediopunkt (Bredel \& Maaß, 2015, S. 65), wie „Bibliotheks-ausweis“ versus „Bibliotheks•ausweis“. Oft wird die zweite Variante mit Mediopunkt bevorzugt, da sie als Sonderform nicht entgegen der Regeln des Standarddeutschen läuft. Der Mediopunkt signalisiert eindeutig die Verwendung von Leichter Sprache, wohingegen der Bindestrich im Standarddeutschen anderen Regeln folgt („Benutzer-ausweis“ vs. „BenutzerAusweis"). Der Bindestrich macht eher da Sinn, wo er ohnehin im Standarddeutschen vorkommt, z. B. „DVD-Regal“ (Bredel \& Maaß, 2015, S. 134).

Ursula Bredel und Christiane Maaß (2015) stellen in ihrem Werk zur Leichten Sprache detailliert vor, wie das Übersetzen in Leichte Sprache gelingen kann und welche Fragen vorab zu klären sind. Neben den gängigen Regeln finden sich dort auch Tipps für die konkrete Übersetzungsstrategie: Um einzuschätzen, welche Wörter auch mit weniger Sprachkenntnissen verstanden werden, empfehlen sie beispielsweise auf duden.de, die Häufigkeit der Wörter zu prüfen (Bredel \& Maaß, 2015, S. 106). Zum Thema gendergerechte Sprache wird empfohlen, abzuwägen, ob andere Regeln damit unterlaufen werden (Bredel \& Maaß, 2015, S. 124 f.). Statt zu schreiben „Lehrer und Lehrerinnen“ ist ihre Empfehlung, am Anfang darauf hinzuweisen: „Im Text stehen immer nur Wörter für Männer. Dann kann man den Text leichter lesen.“ (Bredel \& Maaß, 2015, S. 127) Auch auf das Layout wird eingegangen: Beispielsweise wird eine serifenlose Schrift in mindestens Größe 14 empfohlen. Als goldene Regel für die Bilderauswahl empfehlen Bredel und Maaß (2015): „Wählen Sie nur Bilder mit klaren Aussagen und eindeutigem Text-Bild-Bezug [...].“ (S. 204) Kellermann (2014) fügt hinzu: „Farben sind eher sparsam einzusetzen. Einfache Illustrationen sind besser als Fotos, auf denen zu viele Details zu sehen sind.“ (S. 7) 
Als wichtiges Qualitätsmerkmal gilt, die Texte in Leichter Sprache durch Vertreter^innen der primären Zielgruppe prüfen $\mathrm{zu}$ lassen: Das Logo Leichte Sprache zertifiziert nur solche Übersetzungen, bei denen eine Gegenprüfung stattgefunden hat (vgl. Mammel, 2018, S. 201). Es gibt unterdes noch weitere Qualitätssiegel und Zertifizierungen für Leichte Sprache: So vergibt die Universität Hildesheim ein „wissenschaftlich geprüftes“ Urteil zur jeweiligen Übersetzung, wobei der Verein Inclusion Europe nach einer Prüfung durch Vertreter ${ }^{\star}$ innen der Zielgruppe das bekannteste Siegel „Leichte Sprache“ vergibt (Bredel \& Maaß, 2015, S. 71f.).

\section{Einfache Sprache}

Im Gegensatz zur Leichten Sprache gibt es kein einheitliches Regelwerk für Einfache Sprache (Kellermann, 2014). Im Prinzip bildet sie das gesamte Spektrum zwischen Leichter Sprache und Standardsprache ab (Bredel \& Maaß, 2015, S. 244). Je nach Adressat*innen-Kreis muss also entschieden werden, welche Vereinfachungen sinnvoll und verständlich sind.

Ziel der Einfachen wie auch der Leichten Sprache ist es, einen Text leichter lesbar zu machen - aber ohne den Inhalt $\mathrm{zu}$ verändern. Im Unterschied zur Leichten Sprache ist bei Einfacher Sprache die Verwendung von Nebensätzen möglich (Kellermann, 2014). Sämtliche Alltagsbegriffe werden als bekannt angenommen, Fremdwörter aber möglichst vermieden. Es muss nicht zwingend nach jedem Satz ein Absatz folgen, aber die Übersichtlichkeit des Textes sollte gewährleistet werden. Einfache Sprache erfordert mehr Lesekompetenz und ist daher für viele Personen ein Mittel, um Sachverhalte verständlich zu vermitteln (vgl. Kellermann, 2014). Einfache Sprache ist also flexibler als Leichte Sprache und dabei vielseitig einsetzbar (Bredel \& Maaß, 2015, S. 244). Gudrun Kellermann (2014) verweist auf die tragende Rolle der Bibliotheken für die Verbreitung des Konzepts:

Der Motor für Einfache Sprache waren neben gesetzgebenden Institutionen vor allem Verbände aus dem Bibliotheks- und Verlagswesen. 1992 wurde die Leser Charta verabschiedet, die das Recht auf Lesen verankert und dessen Bedeutung für die Teilhabe an der Gesellschaft heraushebt. (S. 8)

1999 veröffentlichte die IFLA Richtlinien für sogenanntes Easy-Reader-Material (Thornback, 1999). Ohnehin sei der Erfolg der Medien(-verlage) in Leichter Sprache grundlegend auf die Bibliotheken als Hauptkund*innen angewiesen, so die Autor^innen der Richtlinie; zum einen finanziell, was die Verkaufszahlen an- 
geht, zum anderen auch in den Bemühungen, genau diese Menschen zum Lesen zu bringen, die eigentlich Schwierigkeiten damit haben. Als Erfolgsfaktoren werden in der Richtlinie genannt: Netzwerkarbeit mit (Institutionen der) Zielgruppen inklusive einer Bedarfsanalyse für die (potenzielle) Nutzer`innenschaft. Auch die Aus- und Fortbildung der Bibliotheksmitarbeiter*innen ist wichtig sowie ein*e oder mehrere speziell geschulte Lektor*innen für den Bereich. Außerdem sollten die Medien in der Bibliothek leicht auffindbar und eindeutig markiert werden (Thornback, 1999). ${ }^{3}$ Wichtig in der individuellen Beratung ist laut der Richtlinien auch:

Nicht alles Easy-Reader-Material kann jeden Leser in allen Zielgruppen ansprechen. Für ein erfolgreiches Leseerlebnis sind das persönliche Interesse und die Erfahrungen des Lesers fast genauso wichtig wie die Lesbarkeit und die Verständlichkeit des Textes. (IFLA, 1999)

\section{Kritik und Fazit}

Öffentliche Bibliotheken stehen vor der Herausforderung, wie sie kulturelle und Bildungsteilhabe auch für Menschen mit geringer Lese- und Schreibkompetenz in Deutsch ermöglichen können. Mammel (2018) schreibt dazu:

Dies wird über die Bereitstellung eines Buchbestandes in Leichter/Einfacher Sprache hinausgehen müssen: Schriftverkehr/Mahnschreiben bis hin zu Bibliothekssatzungen und Bibliotheksführern in leicht verständlicher Sprache, entsprechende Veranstaltungen oder Sprachkurse bieten hier ein zukunftsträchtiges Aufgabenfeld. (S. 203)

Blickt man auf die Herausforderungen, die mit dem Gebrauch mehrsprachiger Kommunikationsmittel und einem vielsprachigen Bestandsaufbau einhergehen, so bietet Leichte Sprache eine gute Alternative für Bibliotheken. Menschen, deren Muttersprache nicht Deutsch ist, bekommen so die Möglichkeit, Texte einfacher zu verstehen. Allerdings kann Leichte Sprache m.E. nicht als Ersatz für Mehrsprachigkeit dienen, sondern als Ergänzung. Das Angebot von mehrsprachigen Medien und Texten dient nicht allein dem besseren Verständnis, sondern letztlich auch einem respektvollem Umgang mit den sprachlichen Ressourcen der Nutzer^innen. Eine diversitätssensible Ansprache bzw. Kommunikation in der (evtl. zweiten) Herkunftssprache wird oft als wertschätzend und ein „deutliches Willkommen“ gewertet (Allmanritter, 2017, S. 290 f.). Außerdem ist

3 Allerdings kann genau das auch für einige Leser^innen zur Barriere werden, wenn sie sich nicht als auf Leichte Sprache angewiesen „outen“ wollen oder den Easy-Readern ein verstaubtes Image anwohnt. 
$\mathrm{zu}$ beachten, dass viele Menschen mit Migrationshintergrund fließend mehrsprachig sind. Das heißt, sie sind nicht auf Leichte Sprache angewiesen und eine automatische Ansprache zum Thema Easy Reader o. Ä. ist unangebracht. Statt diskriminierende Vorurteile zu verfestigen, muss sensibel über einen respektvollen Umgang reflektiert werden. Das gilt auch, wenn Menschen in die Bibliothek kommen, die (noch) gar kein Deutsch können - denn dann hilft auch keine Leichte Sprache. Dabei ist nicht nur an Geflüchtete zu denken, sondern auch an bspw. Gastwissenschaftler`innen, Tourist*innen oder Austauschschüler`innen.

Das Spannungsfeld zwischen Wertschätzung und Stigmatisierung beschreibt Seitz (2014) wie folgt:

Leichte Sprache, das wird hier deutlich, sorgt somit einerseits für Teilhabe, geht aber mit der Zuschreibung an das Gegenüber einher, auf Leichte Sprache angewiesen zu sein und unterstellt damit ein Defizit. Beides gewinnbringend zusammenzuführen und mit Wertschätzung zu unterlegen, etwa über selbstdifferenzierende Texte, stellt derzeit noch ein konzeptionelles Desiderat dar. (S. 4)

Bei aller Euphorie über den vielfältigen Nutzen der Leichten Sprache als Kommunikationsform, muss man jedoch auch ihre Grenzen beachten (Seitz, 2014, S. 5): Es kann nicht alles eins-zu-eins wie in der Standardsprache ausgedrückt werden. Oftmals sind eine starke Reduktion und wenig Varianz bei den Formulierungen notwendig. Die Schwierigkeit der Leichten Sprache liegt darin, „komplexe Zusammenhänge in klaren Worten und kurzen Sätzen auszudrücken, ohne dass der Sinn verstellt wird oder möglicherweise bevormundend wirkt“ (Seitz, 2014, S. 5). Es handelt sich um eine anspruchsvolle didaktische Arbeit, um das möglichst professionelle Übersetzen von Standardsprache in eine in sich schlüssige, klare und verständliche neue Form.

Gerade in Bibliotheken finden sich unzählige Möglichkeiten, Leichte Sprache zu nutzen und anzubieten: von der Benutzer*innenordnung über die Beschilderung im Haus, die Website, bei Einladungen zu Veranstaltungen, wie auch bei Formularen für die Anmeldung oder in Mahnbriefen. In der ErnstAbbe-Bücherei Jena wurden im Rahmen des $360^{\circ}$-Programms eine Weiterbildung zum Thema organisiert und im Nachgang einige Übersetzungen in Leichte oder Einfache Sprache durchgeführt, bspw. für Informationen zur Benutzer^innenordnung das Angebot von „Schnupperausweisen“ und Informationsflyer zu den interkulturellen Angeboten der Bibliothek. Eine kritische Auseinandersetzung mit Fachjargon, Beratungssprache und sonstiger Kommunikation in der Bibliothek soll auch in der Ernst-Abbe-Bücherei Jena stetig fortgesetzt werden.

Wichtig für den konkreten Einsatz: Die verantwortlichen Mitarbeitenden müssen ausreichend geschult werden (und üben, üben sowie üben). Dabei 
bleibt die Rücksprache mit Expertinnen essenziell. Größere Veröffentlichungen sollten möglichst in Zusammenarbeit mit Vertreter^innen der jeweiligen Zielgruppe erstellt werden. Der Dialog in der Kommunikation mit den Nutzer`innen der Bibliothek bleibt - ob schwere oder leichte Sprache - ohnehin essenziell für eine gute Bibliotheksarbeit.

\section{Literaturverweise}

Aichele, V. (2014). Leichte Sprache: Ein Schlüssel zur „Enthinderung“ und Inklusion. In Politik und Zeitgeschichte, 9-11. S. 19-25.

Allmanritter, V. (2017). Audience Development in der Migrationsgesellschaft: neue Strategien für Kulturinstitutionen. Transcript.

Bredel, U. \& Maaß, C. (2015). Leichte Sprache: theoretische Grundlagen, Orientierung für die Praxis. Duden Verlag.

Bibliotheksportal. (o. J.). Interkulturelle Bibliotheksarbeit. https://bibliotheksportal.de/res sourcen/management/zielgruppen/interkulturelle-bibliothek/ (10.8.2021).

Bundesministerium für Arbeit und Soziales in Zusammenarbeit mit dem Netzwerk Leichte Sprache. (2014). Leichte Sprache. Ein Ratgeber. https://www.gemeinsam-einfachmachen.de/SharedDocs/Downloads/DE/AS/UN_BRK/LS_EinRatgeber.pdf;jsessio nid=D4239D769F02F55CEBDC040FD1091500.2_cid508?_blob=publicationFile \&v=4 (14.2.2021).

Deutscher Bibliotheksverband e. V. (2011). Bibliotheken und die Diversität in der Gesellschaft. Positionspapier von dbv und CILIP. Berlin. https://www.bibliotheksverband.de/filead $\mathrm{min} /$ user_upload/Kommissionen/Kom_IntBib/Stellungnahme_dbv-cilip_Interkulturelle_ Bibliotheksarbeit.pdf (4.9.2020).

Deutscher Bibliotheksverband e. V. (2017, 5. April). Bibliotheksportal: Interkulturelle Bibliothek (Bibliotheksportal). https://bibliotheksportal.de/ressourcen/management/zielgruppen/ interkulturelle-bibliothek (14.6.2021).

Egloff, B., Grosche, M., Hubertus, P. \& Rüsseler, J. (2011). Funktionaler Analphabetismus im Erwachsenenalter: eine Definition. In Projektträger im Deutschen Zentrum für Luft- und Raumfahrt e. V. (Hrsg.), Zielgruppen in Alphabetisierung und Grundbildung Erwachsener. Bestimmung, Verortung, Ansprache: wbv, S. 11-31.

Fachstelle Interkulturelle Öffnung, AWO Regionalverband Mitte-West-Thüringen e. V. (2017). Leichte Sprache. Eine Handreichung für die Praxis. http://www.fs-ikoe.de/neue-handrei chung-leichte-sprache/ (10.8.2020).

Grotlüschen, A., Buddeberg, K. u. a. (2019). LEO 2018 - Leben mit geringer Literalität. Pressebroschüre. Hamburg 2019. https://www.bmbf.de/files/2019-05-07\%20leo-Presseheft_ 2019-Vers10.pdf (8.9.2020).

Hotze, S. (2021). Inklusive Sprache in der Online-Kommunikation von Bibliotheken. In BuB, 02-03. S. 98-101.

International Federation of Library Associations and Institutions (übers. Antje Cockrill). (1999). Richtlinien für Easy-Reader-Material. Den Haag: IFLA Zentrale. https://archive.ifla.org/ VII/s9/nd1/iflapr-57g.pdf (4.9.2020). 
Kellermann, G. (2014). Leichte und Einfache Sprache: Versuch einer Definition. In Aus Politik und Zeitgeschichte, 9-11, S. 7-10.

Mammel, D. (2018). Einfach Lesen! Inklusion und Teilhabe mit Leichter Sprache. In Buch und Bibliothek, 70, S. 200-205.

Nickel, S. (2014). Funktionaler Analphabetismus: Hintergründe eines aktuellen gesellschaftlichen Phänomens. In Aus Politik und Zeitgeschichte, 9-11, S. 26-32.

Rösch, H. (2013). Öffentliche Bibliotheken und ihre Umwelt. In Handbuch Bestandsmanagement in Öffentlichen Bibliotheken. Gantert, K. \& Junger, U. (Hrsg.), München: De Gruyter, S. 7-25.

Seitz, S. (2014). Leichte Sprache? Keine einfache Sache. In Aus Politik und Zeitgeschichte, 911, S. 3-6.

Stefanowitsch, A. (2014). Leichte Sprache, komplexe Wirklichkeit. In Aus Politik und Zeitgeschichte, 9-1, S. 11-18.

Verwaltungsverfahrensgesetz. (2019). Bundesrepublik Deutschland. In der Fassung der Bekanntmachung vom 23.01.2003 (BGBl. I S. 102) zuletzt geändert durch Gesetz vom 21.06.2019 (BGBI. I S. 846) m. W. v. 01.11.2019

\section{Weiterführende Literatur}

\section{Institutionen und Organisationen}

Das Netzwerk Leichte Sprache e. V. gibt es seit 2006. Der Verein verbindet deutschsprachige Prüfer*innen und Übersetzer*innen aus fünf Ländern. Mehr Informationen unter: www. leichte-sprache.org

Eine wissenschaftliche Perspektive bietet die Forschungsstelle Leichte Sprache der Universität Hildesheim. Mehr Informationen unter: www.uni-hildesheim.de/leichtesprache

Weitere Materialien und Tipps stellt das Büro für Leichte Sprache der Lebenshilfe Bremen e. $V$. - inkl. eines Bilder-Katalogs. Mehr Informationen unter: https://lebenshilfe-bremen. de/angebote/buero-fuer-leichte-sprache/

Die Initiative Mensch zuerst des Netzwerk People First Deutschland e. V. bietet weitere Informationen. Mehr Informationen unter: www.menschzuerst.de

\section{Wörterbücher}

Hurraki.de ist ein Online-Lexikon in Leichter Sprache. Hier gibt es freie, kostenlose und zur Weiterverbreitung gedachte Einträge in Leichter Sprache.

Außerdem gibt es das Wörterbuch Leichte Sprache der Bundesvereinigung Lebenshilfe e. V. Dieses ist abrufbar unter: www.lebenshilfe.de/de/leichte-sprache/woerterbuch/

Hilfreich ist auch das Wörterbuch des NDR auf der Homepage des Senders. Mehr Informationen unter: https://www.ndr.de/fernsehen/barrierefreie_angebote/leichte_sprache/ Woerterbuch-in-Leichter-Sprache, hintstartseite100.html 


\section{Verlage}

Der Spaß am Lesen-Verlag in Münster bietet diverse Bücher sowie die Zeitung Klar \& Deutlich. Auf der Seite www.einfachebuecher.de kann man Bücher in Leichter Sprache (A1/A2) und in Einfacher Sprache (A2/B1) bestellen.

Der Passanten Verlag verlegt Literatur in Einfacher Sprache.

Auch im Beltz-Verlag werden Lesetexte in Einfacher Sprache veröffentlicht, unter anderem auch für Kinder. Mehr Informationen unter: superlesbar.de

Edition Naundob aus Berlin ist ein weiterer Verlag in dem Bereich. Mehr Informationen unter www.naundob.de

Außerdem gibt es die Leichter Lesen-Reihe des Ravensburger Verlags mit Geschichten für Kinder in Leichter Sprache.

\section{Sonstiges}

In der Bibliothek des Instituts für Menschenrechte finden sich viele Materialien und Links.

Mehr Informationen unter: institut-fuer-menschenrechte.de/unsere-bibliothek/

Das Bundesministerium für Arbeit und Soziales hat zusammen mit dem Klett-MINT-Verlag Publikationen zu Themen rund um Sozialpolitik in Leichter Sprache und Gebärdensprache erstellt. Mehr Informationen unter: sozialpolitik.com

Zudem gibt es die Nachrichten des Deutschlandfunks in Leichter Sprache. Mehr Informationen unter: nachrichtenleicht.de

Alles rund um Politik in Einfacher Sprache bieten Hefte und Hörbücher der Bundeszentrale für Politische Bildung. Mehr Informationen unter: bpb.de/shop/lernen/einfach-politik/

Interessant sind auch Projekte in/von Bibliotheken, wie beispielsweise die LEA Leseclubs - Lesen Einmal Anders, die bereits in ganz Deutschland stattfinden. Mehr Informationen unter: http://www.kubus-ev.de/lea-leseklub/klubs-deutschlandweit.html 